\title{
Comprehensive Analysis of CRISPR/Cas9-Mediated Mutagenesis in Arabidopsis thaliana by Genome-Wide Sequencing
}

\author{
Wenjie Xu ${ }^{1,2} \oplus$, Wei Fu ${ }^{2}$, Pengyu Zhu ${ }^{2}$, Zhihong $\mathrm{Li}^{1}{ }^{1}$, Chenguang Wang ${ }^{2}$, Chaonan Wang ${ }^{1,2}$, \\ Yongjiang Zhang ${ }^{2}$ and Shuifang Zhu ${ }^{1,2, *}$ \\ 1 College of Plant Protection, China Agricultural University, Beijing 100193 China \\ 2 Institute of Plant Quarantine, Chinese Academy of Inspection and Quarantine, Beijing 100176, China \\ * Correspondence: zhusf@caiq.org.cn
}

Received: 9 July 2019; Accepted: 21 August 2019; Published: 23 August 2019

\begin{abstract}
The clustered regularly interspaced short palindromic repeats (CRISPR)/CRISPR-associated protein (Cas) system has been widely applied in functional genomics research and plant breeding. In contrast to the off-target studies of mammalian cells, there is little evidence for the common occurrence of off-target sites in plants and a great need exists for accurate detection of editing sites. Here, we summarized the precision of CRISPR/Cas9-mediated mutations for 281 targets and found that there is a preference for single nucleotide deletions/insertions and longer deletions starting from $40 \mathrm{nt}$ upstream or ending at $30 \mathrm{nt}$ downstream of the cleavage site, which suggested the candidate sequences for editing sites detection by whole-genome sequencing (WGS). We analyzed the on-/off-target sites of 6 CRISPR/Cas9-mediated Arabidopsis plants by the optimized method. The results showed that the on-target editing frequency ranged from $38.1 \%$ to $100 \%$, and one off target at a frequency of $9.8 \%-97.3 \%$ cannot be prevented by increasing the specificity or reducing the expression level of the Cas 9 enzyme. These results indicated that designing guide RNA with high specificity may be the preferred factor to avoid the off-target events, and it is necessary to predict or detect off-target sites by WGS-based methods for preventing off targets caused by genome differences in different individuals.
\end{abstract}

Keywords: Arabidopsis thaliana; CRISPR/Cas9; off target; whole-genome sequencing

\section{Introduction}

The clustered regularly interspaced short palindromic repeats (CRISPR)/CRISPR-associated protein (Cas) system is a bacterial immune system, which combats invading foreign DNA [1,2], and the type II CRISPR/Cas9 system is the most widely used genome editing tool [3-10] because of its simplicity, efficiency, and user-friendliness [11]. CRISPR/Cas9 technology outperforms traditional plant breeding techniques with an unprecedented genome editing precision and efficiency, allowing accurate modification of agronomical traits without any exogenous fragment by genetic segregation. As a new breeding technology, it has been successfully applied in various species [12,13], including blast-resistant rice [14], drought-tolerant maize [15], and browning-resistant mushroom [16]. It provides unprecedented opportunities for molecular breeding applications.

The CRISPR/Cas9 system is composed of a customized single guide RNA (sgRNA) and a Cas9 enzyme, which recognize the target DNA along with the protospacer adjacent motif (PAM, NGG for Streptococcus pyogenes) through, approximately, a 20 nucleotide (nt) base-pairing interaction [17]. The double-strand break (DSB) generated by Cas9 activates the nonhomologous end joining (NHEJ) or homology-directed repair (HDR) pathway, causing the knockout or replacement of the target gene. 
Compared with HDR, the editing efficiency of NHEJ is higher, and it mainly causes random indels of small fragments at the target site. A few studies have found that this system can induce structural variations (SVs) [18]. However, studies have shown that if the PAM is NAG, there is still high editing efficiency $[19,20]$, and sites with up to six base mismatches aligning to the on-target sequence can also be cleaved by the Cas9 of Streptococcus pyogenes (SpCas9) [21]. Moreover, single nucleotide variations (SNVs) can also impact the specificity of Cas9. Yang et al. found that a single base genetic variation produced a new off-target site with high editing efficiency in human-induced pluripotent stem cells [22]. Studies of animals showed that off-target activity can be reduced by using improved engineered Cas 9 variants $[23,24]$ and controlling the enzyme concentration [25-27], but there is little evidence to prove that and few off targets were found in plants [28].

Human-desired variations induced by CRISPR/Cas9 system are not spontaneous variations, and unexpected editing events may be a safety hazard for CRISPR/Cas9-mediated crops [29,30]. Whole-genome sequencing (WGS) has become the preferred method for detecting genome-edited mutations because it provides comprehensive information about genomic variations, including on-/off-target variations [31,32]. Feng et al. and Peterson et al. detected off targets in the T1 and T2 genome-edited mutants of Arabidopsis by WGS and found no off-target sites [33,34]. Tang et al. conducted a WGS analysis of 34 plants edited by Cas 9 in the T0 and T1 generations, along with three types of control plants in Oryza sativa Nipponbare. It was found that the SNVs and indels identified in edited T0 plants are largely background mutations [35]. Similarly, Li et al. demonstrated that the CRISPR/Cas9-edited plants had few off-targets in cotton, which contains a complex and large genome, and the differences between the offspring of the same parent or the number of somatic mutations during tissue culture were much greater than the number of CRISPR/Cas9-induced off-target sites. However, the study also found that 61 potential off-target sites and PAM were generated by genetic variations in wild-type (WT) plants [36]. Designing sgRNA with high specificity by software [37-40] is the primary method for plant breeders to reduce off-targets.

However, the sequencing depth of WGS is limited, and most of these studies detected off-targets focusing on either predictable off-target sites with a small number of base mismatches or 20-25 nt flanking of these sites, which may lead us to ignore some low-frequency off-targets. This evidence may be insufficient to prove that we can ignore the off-target effect in plants. Actually, many WGS-based methods have been developed to detect or predict off-target sites, including GUIDE-seq [41], Digenome-seq [42], and CIRCLE-seq [21]. These methods have been widely used for off-target detection in animals and results showed that the CRISPR/Cas9 system is highly specific or has varying degrees of off-target effects $[23,43]$. However, there have been few such applications in plant research, and there is limited evidence for the common occurrence of off-target sites.

Although genome editing in plants is different from gene therapy, mutants without off-target or exogenous fragments can be obtained by backcrossing and selfing, but this process is time consuming and laborious, which will slow down the process in crop breeding. In addition, unpredictable off-target effects attract the attention of the public and regulatory authorities, and there is no unified international regulatory strategies [29]. Therefore, it is necessary to reduce or avoid off-target incidence and accurately examine the molecular characteristics of genome editing plants. Here, we suggested a method for whole-genome detection of editing sites in CRISPR/Cas9-mediated plants by summarizing the precision of on-target editing for more than 10 species. In addition, combining with Digenome-seq and targeted amplification deep sequencing, we examined the influence of specificity and expression level of Cas9 on on-/off-target editing in Arabidopsis thaliana.

\section{Results}

\subsection{Summary of On-Target Variations in CRISPR/Cas9-Edited Plants}

In recent years, studies on the detection of gene editing sites by WGS have increased, but there is no clear criterion for identifying small and midsize indels which may be ignored. To capture 
editing mutations more accurately, we collected the on-target mutations of genome-edited plants in 95 published articles, including more than 10 species, such as Arabidopsis, rice, cotton, maize, soybean, and wheat, covering 195 genes and 281 targets. According to the on-target mutations given in the publications, the indel size and the location distribution of all variations were summarized.

The analysis results showed that single nucleotide indels were the most common mutations for the majority of targets (Figure 1A). The insertion positions were mainly located at the cleavage sites (commonly $3 \mathrm{nt}$ upstream of PAM) or $1 \mathrm{nt}$ upstream (Figure 1B). Compared to insertions, more targets showed a preference for longer deletions (up to $40 \mathrm{nt}$ ), which mainly started from $40 \mathrm{nt}$ upstream to $5 \mathrm{nt}$ downstream of the cleavage site (Figure 1C), and the ending positions extended to $30 \mathrm{nt}$ downstream of the cleavage site (Figure 1D). Based on the results, we determined that the sequences $50 \mathrm{nt}$ upstream and $50 \mathrm{nt}$ downstream of the mutations would be extracted as candidate sequences for editing sites-detection by WGS.

(A)

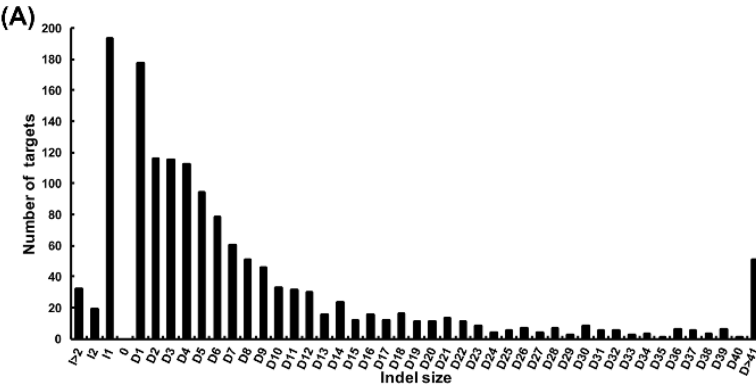

(C)

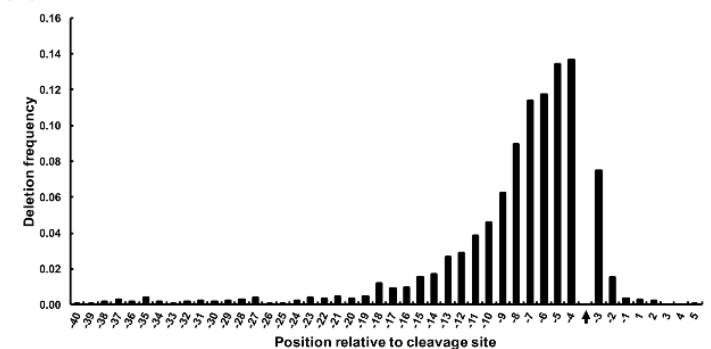

(B)

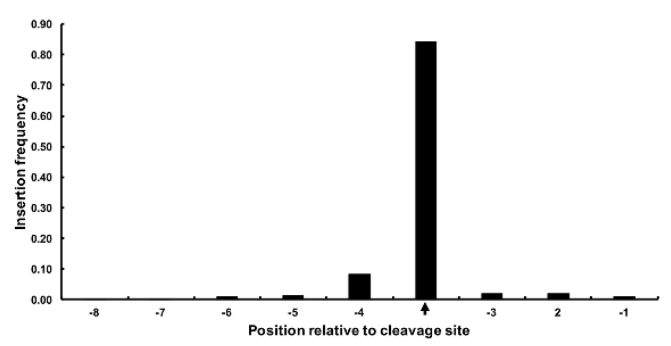

(D)

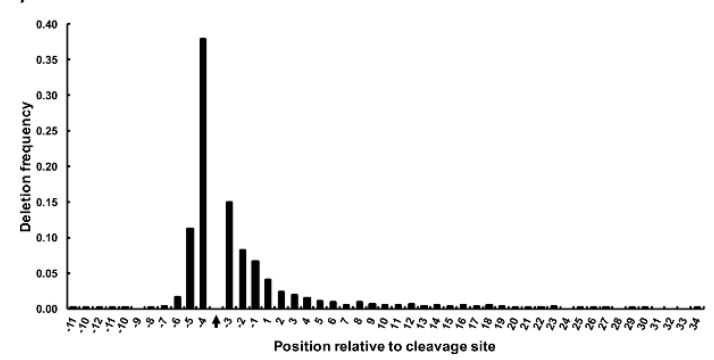

Figure 1. Summary of on-target variations in multiple plants subjected to clustered regularly interspaced short palindromic repeats (CRISPR)/CRISPR-associated protein (Cas9)-mediated editing. (A) Distribution of the most common indel size at on-target sites. ' $\mathrm{D}$ ' represents deletion and ' $\mathrm{I}$ ' represents insertion. (B) Distribution of the insertion sites of the targets. ' -1 ' on the $X$-axis represents $1 \mathrm{nt}$ upstream of the protospacer adjacent motif (PAM), and black arrows represent cleavage sites. (C) Distribution of the starting sites of deletions at target sites. (D) Distribution of the ending sites of deletions at target sites.

\subsection{Off-target Sites Predicted by Digenome-seq}

zCas9 is a maize codon-optimized SpCas9 mutant with high on-target editing efficiency, but its off-target effect has not been fully evaluated. To detect the DNA targeting specificity of the zCas9 and SpCas9 enzymes in vitro and shorten the number of potential off-target sites, we performed Digenome-seq to capture the cleavage sites of SpCas9 and zCas9. For all 10 target sequences, there were at least 10 base mismatches between each pair of them. The SpCas9/sgRNA ${ }^{-}$control sample was sequenced to a depth of $45 \times$, and the SpCas $9 / \mathrm{sgRNA}^{+}$and zCas9/sgRNA ${ }^{+}$experimental samples were sequenced to a depth of $70 \times$. The genome mapping ratio of the $\mathrm{zCas} 9 / \mathrm{sgRNA}{ }^{+}$sample was $93.72 \%$, but the SpCas9/sgRNA ${ }^{-}$sample and the SpCas9/sgRNA ${ }^{+}$sample were both lower than $80 \%$, which may be caused by random DNA degradation by different enzymes.

Studies have shown that sequences with 6 mismatches mapping to the targets may also be cut by the CRISPR/Cas9 system, so to avoid false positives, we selected potential off-target sites with 
zero to six mismatches or one bulge with no mismatch. As expected, the SpCas9/sgRNA ${ }^{-}$control sample did not detect any cleavage sites. SpCas9/sgRNA ${ }^{+}$and zCas9/sgRNA ${ }^{+}$samples identified 51 and 16 sites separately, and all off-target sites of zCas $9 / \mathrm{sgRNA}^{+}$were included in the SpCas9/sgRNA ${ }^{+}$ result (Figure 2A). Although the samples had different degrees of DNA degradation, the prediction results of Digenome-seq were still credible. These off-targets were classified according to the number of mismatches. There were only four off-targets with three to six mismatches for $\mathrm{zCas} 9 / \mathrm{sgRNAs}{ }^{+}$, and the number of cleavage sites was less than SpCas9/sgRNAs ${ }^{+}$, which was 39 sites (Figure 2B). The results indicated that in vitro cleavage specificity of zCas9 was significantly higher than SpCas9. Compared to the prediction results of Cas-OFFinder using the common criteria, which obtained 29 editing sites with zero to three mismatches or one bulge with zero mismatches of the 10 targets, 14 sites were intersected with the Digenome-seq results of zCas9/sgRNAs ${ }^{+}$, and 18 sites intersected with the SpCas9/sgRNAs ${ }^{+}$ results (Figure 2A). We increased the mismatches to five to predict off-targets by the tool (Table 1), and the comparison results showed that predicting off-targets by Digenome-seq effectively reduced the number of potential off-target sites of each target.

(A)

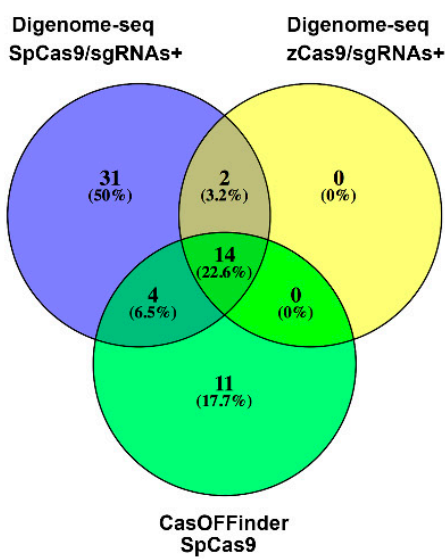

(B)

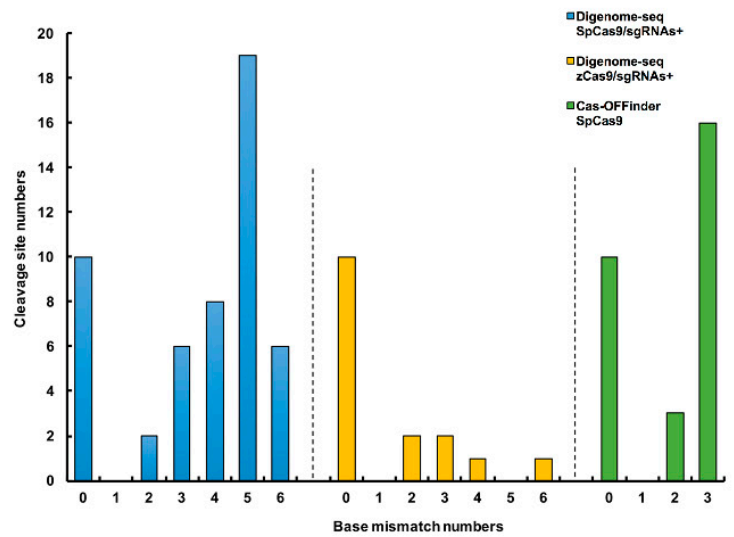

Figure 2. Comparison of off-target prediction results. (A) Venn diagram of predicted off-target sites of Digenome-seq and Cas-OFFinder. (B) Distribution of base mismatches with on-target sequences of digenome-seq and Cas-OFFinder. The number of mismatches did not include mismatches in the PAM sequence.

Table 1. Number of off-target sites predicted by Digenome-seq and Cas-OFFinder.

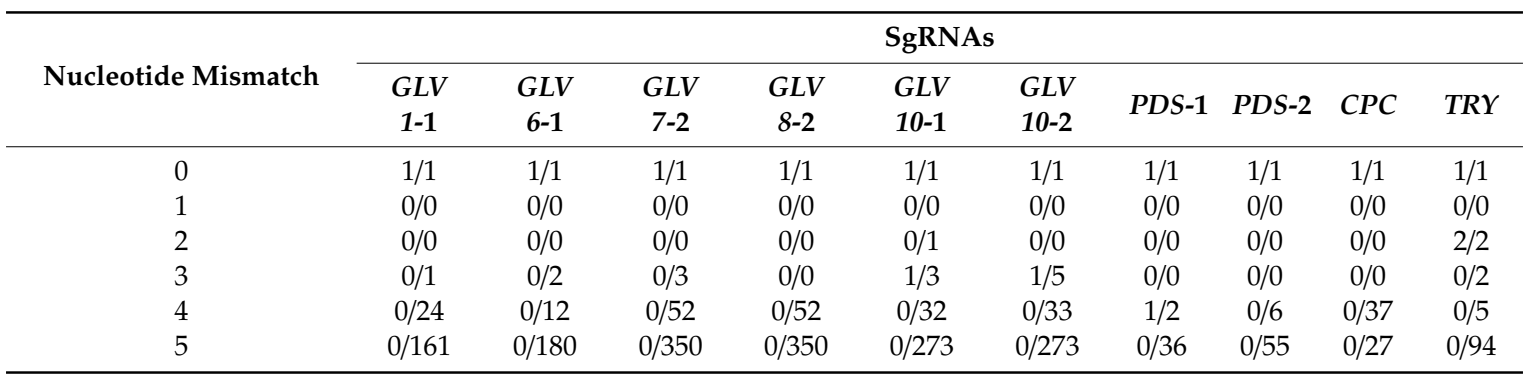

Predicted off-target number within 5 mismatches: The number of mismatches did not include mismatches in the PAM sequence. The number in front of the ' $/$ ' represents the off targets predicted by Digenome-seq and number behind the ' $/$ ' represent the off targets predicted by Cas-OFFinder tool.

\subsection{Detection of Genome-Wide Variations and Editing Sites}

To further study the off-target effects of genome-edited plants, we constructed TRY/CPC and PDS-1/PDS-2 double mutants with pHSE401 and pHEE401E expression vectors. TRY and CPC are two genes that negatively regulate Arabidopsis epidermal hair-specific expression genes, and their knockout will cause leaves to form clustered leaf trichomes [44]. The PDS gene is a chlorophyll synthesis gene, and the mutants show an albino phenotype [45]. After Sanger sequencing of the antibiotic-screened T1 
plants with obvious phenotypes, we obtained TRY/CPC double mutants with two different vectors (TC/TCE) and PDS-2 single mutants with the pHEE401E vector (PDSE) (Figure 3). Six mutants were selected for WGS, including PDSE-3, PDSE-12, TC-15, TC-31, TCE-31, and TCE-42. In addition, three same-generation WT plants of T0 were also included for WGS as control samples to filter spontaneous mutations. All mutants were sequenced with an average of $95 \times$ to $140 \times$, and the average sequencing depth of the three WT plants was $80 \times$ to $90 \times$ (Table S1).

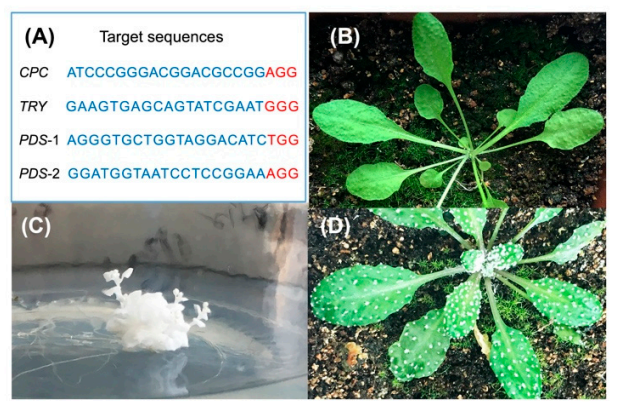

Figure 3. Arabidopsis T1 mutants obtained via CRISPR/Cas9. (A) Target sequences for constructing CRISPR/Cas9-edited mutants. The $19 \mathrm{nt}$ target sequences and the following PAMs (NGG) are highlighted in blue and red, separately. (B) The phenotype of wild-type Arabidopsis. (C) The phenotype of CRISPR/Cas9-edited PDS mutant. (D) The phenotype of CRISPR/Cas9-edited TRY and CPC mutant.

First, we identified the T-DNA insertion events. PDSE-3, TC-15, TCE-31, and TCE-42 had only one T-DNA insertion, but both the PDSE-12 and TC-31 mutants had multiple-copy insertions (Figure S1). Then, genome-wide variations were analyzed basing on the pipeline in Figure $4 \mathrm{~A}$. To eliminate the influence of the genetic background on the subsequent analysis, the genomic variations of each mutant were subjected to WT variation filtering. There were 608 and 612 SNVs in PDSE-3 and TC-15 mutants, respectively, which were significantly more than other mutants (Table 2). The $573 \mathrm{SNVs}$ of the two mutants were consistent but had no overlap with the SNVs of the other mutants and most of these SNVs were homozygous or heterozygous, which indicated that they may be heritable spontaneous variations from parents. These results suggested that there may be great differences in the genomes of same-generation individuals.

In order to reduce the effects of Agrobacterium transfection, different parental genomic differences, sensitivity of software, and variations caused by other factors in each mutant were identified by deducting the variations from mutants with different target sites. The genomic variations of each mutant were significantly reduced, and the number of SNV variants of PDSE- 3 and TC-15 were similar to those of the other mutants (Table 2; Figure 4B,C). Further analysis of the genome-wide distribution of these variations in these mutants revealed no hot spots (Figure 4D). For the on-target sites of all mutants, the editing efficiency was $38.1 \%-100.0 \%$ (Table 3). Surprisingly, all TRY/CPC double mutants had one identical off-target of TRY, with two mismatches located in CPC gene, and the mutation frequency ranged from $9.8 \%$ to $97.3 \%$. The TC-15 mutant had one variation annotated in the repeated sequence with six mismatches of the TRY target, which was not predicted by Digenome-seq, so this variation was not a bona fide off-target mutation. We used the same analysis method to reanalyze the published WGS data for the other six sgRNAs in the Digenome-seq and detected all on-target mutations, but no off-target was found. We also detected the complex SVs shorter than $10 \mathrm{~kb}$ of these mutants and no SV was found in any editing sites (Table S2). 
(A)

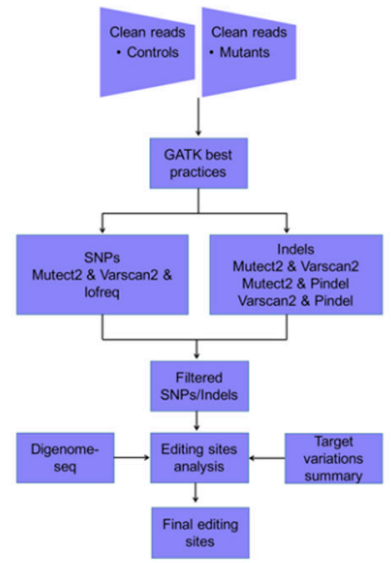

(D)

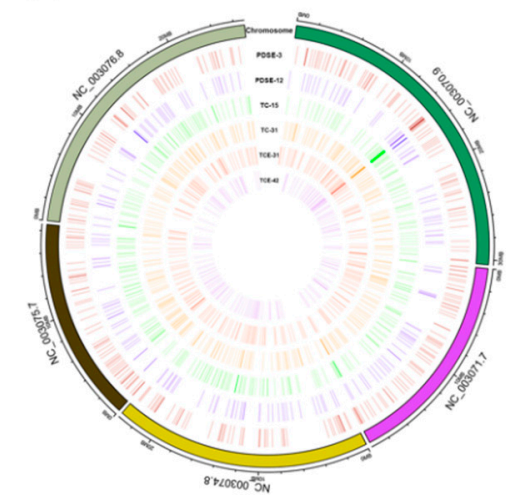

(B)

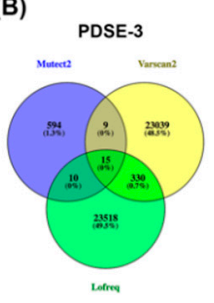

(C)

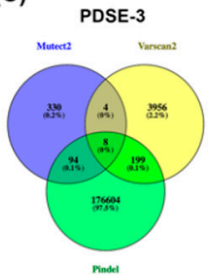

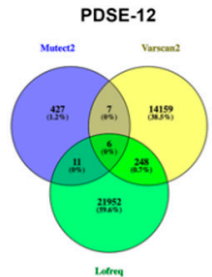

tum

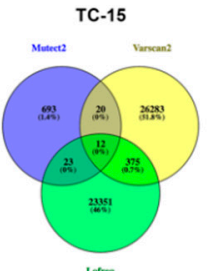

nens

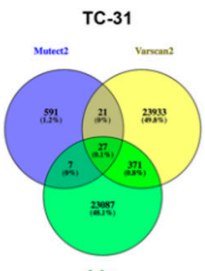

Latreg

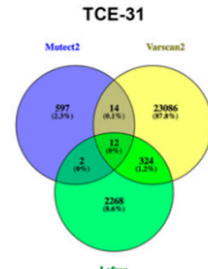

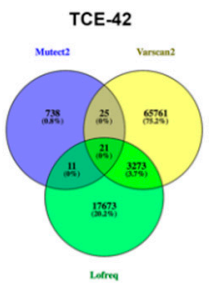

PDSE-12
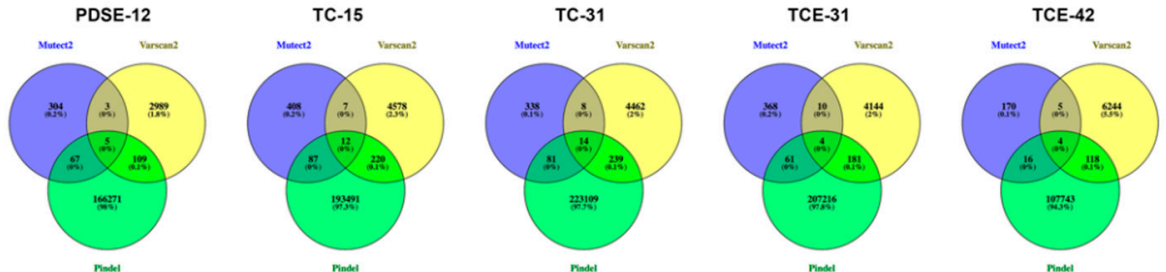

Figure 4. Whole-genome variations analysis in genome-edited mutants. (A) Workflow of whole-genome detection of single nucleotide variations (SNVs) and indels. (B) Venn diagram of SNVs in genome-edited mutants detected by variant callers. (C) Venn diagram of indels in genome-edited mutants detected by variant callers. (D) Genome-wide distribution of mutations in CRISPR/Cas9-edited mutants.

Table 2. Variations in CRISPR/Cas9-edited mutants.

\begin{tabular}{ccccc}
\hline \multirow{2}{*}{ Mutant } & \multicolumn{2}{c}{ SNV } & \multicolumn{2}{c}{ Indel } \\
\cline { 2 - 5 } & WT $^{-}$ & WT $^{-}$/DT $^{-}$ & WT $^{-}$ & WT $^{-}$/DT $^{-}$ \\
\hline PDSE-3 & 608 & 15 & 3120 & 305 \\
PDSE-12 & 10 & 6 & 1968 & 184 \\
TC-15 & 612 & 12 & 2525 & 326 \\
TC-31 & 31 & 27 & 2332 & 342 \\
TCE-31 & 14 & 12 & 2586 & 256 \\
TCE-42 & 24 & 21 & 690 & 143 \\
\hline
\end{tabular}

'WT'- represents variations in mutants filtered with wild-type (WT) plants; 'WT'/DT' represents variations in mutants filtered with WT plants and other different target (DT) variations. Variations of CRISPR/Cas9-edited PDS, $C P C$, and TRY mutants were deducted for each other. 
Table 3. On-/off-target mutation frequency of WGS.

\begin{tabular}{ccccc}
\hline Mutant & PDS-2 (\%) & CPC (\%) & TRY (\%) & TRY Off Target (\%) \\
\hline PDSE-3 & 38.1 & - & - & - \\
PDSE-12 & 46.1 & - & - & - \\
TC-15 & - & 84.1 & 83.5 & 20.9 \\
TC-31 & - & 85.7 & 88.1 & 97.3 \\
TCE-31 & - & 87.6 & 95.3 & 9.8 \\
TCE-42 & - & 100 & 84.2 & 19 \\
\hline
\end{tabular}

\subsection{Analysis of Targeted Amplification Deep Sequencing}

Given that leaves are composed of a large number of cells with multiple types, there are various cell types in leaves [34], and multiple editing events may occur in mutants constructed by somatic cell-expressed Cas9. Moreover, low-frequency off-targets may be ignored because of the limited amount of WGS data, so, targeted amplification deep sequencing of PDSE-3; TC-15; TC-31; and pooling samples of WT, PDSE-MIX, TC-MIX, and TCE-MIX were performed to further analyze the editing sites. Except for the PDSE-MIX, which was pooled from 20 mutants, the remaining two samples contained mixtures of five plants. In addition, the zCas9 of TCE mutants was specifically expressed in the egg cell, for which the expression time was shorter than that of TC mutants, and TC mutants with different T-DNA copies had different expression levels of zCas9. Twenty-two sites were subjected to targeted deep sequencing, including all predicted sites of zCas9 by Digenome-seq, off-targets with two to three mismatches predicted by Cas-OFFinder, and randomly selected off-target sites of SpCas9 predicted by Digenome-seq. All sites were sequenced with more than 100,000 reads except the TRY-5 site. The on-/off-target mutagenesis frequencies were calculated as the ratio of reads containing indels in the target sites to the total reads captured.

Mutations with frequencies below $1 \%$ o would not be considered bona fide mutations, as such low-frequency mutations may have resulted from sequencing errors. We calculated the on-target editing efficiency via WGS and deep sequencing in PDSE-3, TC-15, and TC-31 and the Pearson correlation between them was $0.997(\mathrm{p}<0.01)$. Only one off-target site with two mismatches of the TRY target was edited, and the PDS-1 site had no mutations (Table S3; Figure 5A). These results indicated that the deep sequencing results were credible and the whole-genome detection method is effective.

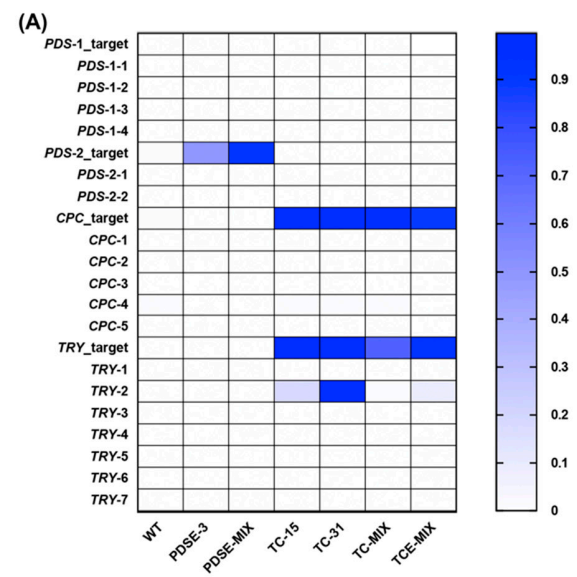

(B)
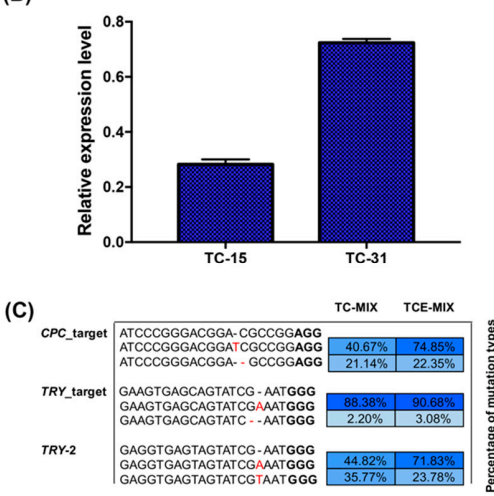

Figure 5. Targeted amplification deep sequencing of genome-edited mutants. (A) Heat map of the editing efficiency of on-/off-target sites. (B) Comparison of the zCas9 expression of TC-15 and TC-31 by RT-qPCR. The relative expression level was calculated by $2^{-\Delta C t}$ method. (C) High proportion of variation types in TC-MIX and TCE-MIX. Deletions and insertions are indicated by "-" and red letters, respectively. 
The CPC and TRY on-target editing efficiency of the TC-31 and TC-15 mutants were above $99.0 \%$, and the off-target efficiency of the TC-31 was equivalent to the on-target sites, which was $98.2 \%$. This result was consistent with the research that off-target editing efficiency may be equal to, or higher than, on-target editing efficiency in human cells [31]. However, the off-target editing efficiency of TC-15 was only $18.6 \%$, indicating that a high expression level of zCas9 may have increased the off-target editing efficiency (Figure 5A,B). Compared with TC-MIX, the editing efficiency of the TRY target and the one inevitable off-target in TCE-MIX were relatively high. Moreover, the main mutation types of TC-MIX and TCE-MIX were the same, indicating that repairing via the NHEJ pathway may have some biases (Figure 5C).

\section{Discussion}

In this study, we first collected the target mutations of more than 10 CRISPR/Cas9-mediated species in plants and summarized the size and location distribution of the most common editing mutations. Finally, sequences $50 \mathrm{nt}$ upstream and $50 \mathrm{nt}$ downstream of mutations, detected by WGS, were considered as candidate sequences to identify editing sites by calculating the number of mismatches. The method is different from the currently used detection methods, which focus on the flanking 20-25 nt of the mutations or the potential off-target sites predicted by tools. To verify the effectiveness of the method, we also reanalyzed the public WGS data of four rice mutants [35] by this method and found more off-target sites within three mismatches (Table S4), indicating that the proposed detection method is effective and more sensitive than that used in the publication.

Compared to the large amount of research on CRISPR/Cas9-induced off-targets in animal cells, there are less studies that found off-targets in plants. Although plant researchers have recently tended to use WGS to detect off-target sites instead of using Sanger sequencing, it is difficult to detect low-frequency off-targets because WGS data are limited. Therefore, based on WGS detection of genome editing mutations, we used targeted amplification deep sequencing to further analyze off-targets with mutation frequencies above $1 \%$. All TRY/CPC double mutants in this study had one identical off-target, which had two mismatches with the TRY target, while the other off-targets with three mismatches and some of the off-target sites predicted by Digenome-seq were not detected. Studies reported that mismatches close to PAM are more critical than mismatches in distal positions [31,46], and Pattanayak et al. suggested that perfect complementarity between sgRNA and target sequences is required in the 7-12 base pairs adjacent to the PAM [47]. Nevertheless, Zhang et al. found that the tolerances to mismatches can occur at any position of the sgRNA [48]. There are differences in the base mismatch complementarity principle between different studies, but the editing efficiency of off-target sites with more than three mismatches is greatly reduced $[46,49]$, so for genome editing plants, most plant breeders focus on off-target sites within three mismatches.

WGS analysis of multiple CRISPR/Cas9 genome-edited mutants of rice showed that the insertion of T-DNA with multiple copy numbers does not induce more off-targets within three mismatches [35]. In this study, the TC-15 and TC-31 mutants had different T-DNA copy number insertions, and there was no difference in the on-target sites. However, the editing efficiency of the off-target site in TC-31 mutants was similar to the on-target efficiency, which was significantly higher than the off-target efficiency in TC-15. This result indicated that a low expression of Cas9 did reduce off-target efficiency, but off-target events could not be completely avoided. Therefore, more attention should be paid to designing sgRNAs with high specificity.

Predicting off-targets by tools is widely accepted by researchers, but different tools have different prediction rules. Some tools predict off-targets by counting the base mismatches and mapping PAM, such as Cas-OFFinder. CHOPCHOP and GT-Scan do not allow mismatches in the seed regions when predicting off-target sites, and some characteristics and parameters can be specified to predict off targets by the Breaking-Cas tool [50]. All tools rely on a known reference genome and only detect predictable off targets. In this study, we found that there were hundreds of SNV differences in different individuals. These heritable spontaneous variations may produce unknown off-target sites that cannot be predicted 
by software. Studies showed that Cas9 can recognize canonical NGG and noncanonical NAG or NGA PAM sequences. There may still be some sites ignored, even though a large number of off-target sites will be obtained by tools. Although we did not find any targets caused by inherent variations in this study, it is still necessary to comprehensively detect on-/off-targeting by WGS-based methods to assess the safety of genome-edited plants at the molecular level. For species with incomplete genome annotation, de novo sequencing of the genome before designing sgRNA to obtain highly specific target sequences may be required.

In addition, the genome editing mutants constructed in this study used two vectors with Cauliflower Mosaic Virus 35S promoter (CaMV 35S) and egg cell-specific promoter, and the deep sequencing results of multiple pooled mutants showed that controlling the expression time of the Cas9 enzyme did not reduce the off-target efficiency. We can avoid complex editing events by selecting appropriate delivery methods for the CRISPR/Cas9 system or developing 'off-switch' techniques [51,52] to terminate the expression of the system at a specific time. Shou et al. found that Cas9-mediated nucleotide insertions are nonrandom and are equal to the combined sequences upstream of both PAM sites [53]. Surprisingly, the main mutation types of TC-MIX and TCE-MIX were identical, which suggested that Cas9-mediated nucleotide insertions are nonrandom and there may be some biases in repairing the DSB. The results may be helpful for us to obtain genome-edited plants with specific variations.

In this study, we comprehensively analyzed the variations in on-target sites and the effects of several factors on off-target events in Arabidopsis. Compared with nonmodel plants, Arabidopsis has a complete genome annotation, and the genome size is only $119.7 \mathrm{Mb}$ (NCBI), which is conducive to clearly revealing the off-target effects of gene editing. Although the number of mutants in this study was limited, the phenomena revealed are useful for the construction of CRISPR/Cas9-edited mutants and the detection of editing sites. To reveal the ubiquitous influence of various factors on CRISPR/Cas9-mediated genome-edited plants, more experimental data may be needed.

\section{Materials and Methods}

\subsection{Summary of Targeting Precision in Genome-Edited Plants}

To collect editing mutations of different target sites and support the detection of off-target variations by WGS, we searched for published CRISPR/Cas9-mediated genome editing articles in PubMed, Science Direct, Elsevier, and other databases with keywords such as "plant", "crop", and "CRISPR/Cas9" in the time range of 2014-2019. Then, we analyzed the size and location distribution of mutations caused by CRISPR/Cas9 system. All publications can be found in the Supplementary Materials.

\subsection{In Vitro Cleavage of Genomic DNA and Cleavage Site Analysis}

SpCas9 protein was purchased from New England Biolabs. zCas9 protein was expressed and purified using a previous method [54]. SgRNAs were synthesized using the MEGAshortscript T7 Transcription Kit (Thermo Fisher Scientific, Vilnius, Lithuania) according to the manufacturer's manual. Transcribed RNA was purified by ethanol precipitation and quantified by spectrometry. SpCas9 or zCas9 protein $(40 \mu \mathrm{g})$ and $10 \mathrm{sgRNAs}(2.7 \mu \mathrm{g}$ each) were preincubated at room temperature for $10 \mathrm{~min}$ to form ribonucleoprotein (RNP) complexes. Genomic DNA $(8 \mu \mathrm{g})$ was incubated with RNP complexes in NEB reaction buffer 3.1 for $6 \mathrm{~h}$ at $37^{\circ} \mathrm{C}$. The digested genomic DNA was treated with RNase A $(50 \mu \mathrm{g} / \mathrm{mL})$ to degrade sgRNAs and purified again with ethanol precipitation. In parallel, genomic DNA without sgRNAs was also processed under the same conditions as a control sample. Then, approximately $1 \mu \mathrm{g}$ of SpCas9/sgRNA ${ }^{+}, \mathrm{zCas} / \mathrm{sgRNA}^{+}$, and SpCas9/sgRNA ${ }^{-}$digested genomic DNA were used for WGS on an Illumina HiSeq X Ten with an average 50× depth at CapitalBio Technology Company (Beijing, China). The identification of sites cut by RNP in vitro was performed as described before [49]. Finally, potential off-targets limited to 6 mismatches mapping to $22 \mathrm{nt}$ on-target sequences were selected for further analysis. 


\subsection{Guide RNA Design and Vector Construction}

The sgRNAs were designed according to the CRISPRscan web tool (https://www.crisprscan.org/ ?page=sequence). The CDS sequences of $P D S, C P C$, and TRY target genes were selected for further analysis. First, we found all possible sgRNA sequences in target genes, and for the top-ranked targets, Cas-OFFinder (http://www.rgenome.net/cas-offinder/) was used to predict the off-target sites. Then, two sgRNAs were designed for the PDS gene (PDS-1 and PDS-2) and one sgRNA each for CPC (CPC) and TRY (TRY). Plasmids encoding zCas9 and two sgRNAs were generated by ligating pHSE401 [44] or pHEE401E [55] with the pCBC-DT1T2 [44] fragment after digestion with BsaI. All these vectors were provided by Prof. Qijun Chen (China Agricultural University). Moreover, we also selected 6 sgRNAs (GLV1-1, GLV6-1, GLV7-2, GLV8-2, GLV10-1, and GLV10-2) from previous reported research for predicting off-target sites in vitro [34]. All target sequences are listed in Table S5.

\subsection{Arabidopsis Transformation and Mutagenesis Analysis at On-Target Sites}

Two expression vectors were transformed into Agrobacterium strain GV3101. WT Arabidopsis Col-0 plants were grown in a growth chamber with constant light at $23^{\circ} \mathrm{C}$. Plants were transformed via the floral dip method. Seeds derived from the T0 plants were screened on MS plates containing $25 \mathrm{mg} / \mathrm{L}$ hygromycin, and the resistant seedlings were transplanted to soil. Then, the genomic DNA of T1 transgenic plants with obvious phenotypes was extracted with the DNeasy Plant Mini Kit (Qiagen, Hilden, Germany). To confirm mutations in PDS, TRY, and CPC, we amplified fragments surrounding the target sites of the three genes by PCR using specific primers (Table S6).

\subsection{Whole-Genome Sequencing and Variation Analysis}

Genomic DNA was extracted from fresh leaves of three WT plants, two mutants for each CRISPR/Cas9 expression plasmid using the DNeasy Plant Mini Kit. For each sample, approximately $2 \mu \mathrm{g}$ of DNA was used for WGS on an Illumina HiSeq X Ten (paired-end $150 \mathrm{bp}$ ) (CapitalBio Technology Company, Beijing, China), with an average 100× sequencing depth for genomic editing plants and $50 \times$ for WT plants at the CapitalBio Technology company. Adapters were trimmed using Cutadapt (v. 1.18) [56]. The WGS data reported in this article are available in the Sequence Read Archive in National Center for Biotechnology (NCBI) BioProject: PRJNA549435.

Cleaned reads were mapped to Arabidopsis reference sequence 10.1 (https://www.ncbi.nlm.nih.gov/) with Bowtie2 (v. 2.2.3) [57] software. The Genome Analysis Toolkit (GATK, v. 3.7) [58] was used to realign reads near indels and recalibrate base quality scores by following GATK best practices (https://software.broadinstitute.org/gatk/best-practices/). Whole-genome SNVs were detected by LoFreq (v. 2.1.0) [59], MuTect2 [60], and VarScan2 (v. 2.3.9) [61], and whole-genome indels were identified using MuTect2, VarScan2, and Pindel (v. 0.2.5b9) [62]. Structural variations were analyzed by Delly (v. 0.7.2) [63] and Pindel. To avoid the adverse effects of sequencing errors and missing low-frequency SNVs and indels, common variations detected with at least a depth of $2 \times$ for each site were retained for further analysis. After filtering spontaneous variations with WT samples, according to the summary of the common size and location distribution of CRISPR/Cas9-mediated mutations in plants, we captured the upstream and downstream sequences of SNVs that occurred repeatedly in the results from three programs and indels that were found by at least two programs for the editing site analysis. Structural variations within $10 \mathrm{~kb}$ detected by both tools were selected for the editing sites identification. Candidate sequences with 0-6 mismatches mapping to $22 \mathrm{nt}$ on-target sequences were compared with the predicted results.

\subsection{Targeted Amplification Deep Sequencing}

Genome-edited and WT plants were used to perform target amplification deep sequencing. Young leaves were used for genomic DNA extraction using the DNeasy Plant Mini Kit. The primers used in the deep sequencing can be found in Table S7. The libraries were purified with a QIAquick PCR 
Purification Kit (Qiagen, Hilden, Germany) and sequenced on the Illumina X-Ten platform. Bowtie2 was used to map the sequencing data to target sequences, and indels with at least $2 \times$ depth were counted as on-target or off-target variations. The ratio of the number of mutant reads to the total number of target reads was used as the editing efficiency.

\subsection{Real-time Quantitative PCR}

Young leaves pooled from $12 \mathrm{~T} 2$ plants of TC- 15 and TC-31 were used to analyzed the expression levels of the Cas9 by performed the real-time quantitative PCR (RT-qPCR). PCR primers specific to these genes were designed using PrimerQuest Tool (https://sg.idtdna.com/Primerquest/Home/Index) (Table S8). The total RNA was extracted using the RNAprep Pure Plant Kit (Tiangen, Beijing, China) and reverse-transcribed using the protocol of cDNA Synthesis SuperMix (TransGen Biotech, Beijing, China). All RT-qPCR experiments were performed with Applied Biosystems 7900 instruments. The PCR consisted of $10 \mu \mathrm{L}$ of FastSYBR Mixture (High ROX), $1 \mu \mathrm{L}$ of forward and reverse primers $(10 \mathrm{pM} / \mu \mathrm{L})$, $7 \mu \mathrm{L}$ of distilled water, and $1 \mu \mathrm{L}$ of cDNA in a total volume of $20 \mu \mathrm{L}$. The thermal cycling conditions were $3 \mathrm{~min}$ at $95^{\circ} \mathrm{C}$ followed by 40 reaction cycles $\left(10 \mathrm{~s}\right.$ at $95^{\circ} \mathrm{C}$ and $20 \mathrm{~s}$ at $\left.60^{\circ} \mathrm{C}\right)$. The gene ACTIN2 was used as the reference gene. The mean quantification cycle value of the triple reactions was used to calculate the relative expression levels of the target genes according to the $2^{-\Delta \mathrm{Ct}}$ method.

\section{Conclusions}

Here, we proposed an accurate method for whole-genome detection of editing sites in CRISPR/Cas9-mediated plants. The results indicated that designing guide RNA with high specificity may be the preferred factor to minimize the possibility of off-targeting, and genome-wide detection of editing sites is inevitable. Our research offered a list of suggestions for how to avoid or reduce the probability of off-target events in plants, which can promote progress in crop improvement by CRISPR/Cas9 system.

Supplementary Materials: Supplementary materials can be found at http://www.mdpi.com/1422-0067/20/17/ 4125/s1.

Author Contributions: S.Z., W.F., and W.X. designed this study. W.X., Chenguang, W., and Chaonan, W. performed the experiments. W.X. analyzed and interpreted the data. W.X. and S.Z. prepared the manuscript. P.Z. and Y.Z. revised the manuscript. W.F. and Z.L. managed the research progress.

Funding: This research was funded by National Science and Technology Major Project, grant number 2018ZX08012001".

Acknowledgments: We thank Qijun Chen (China Agricultural University, Beijing) for kindly providing the plant binary vector $\mathrm{PHSE} 401$ and $\mathrm{pHEE} 401 \mathrm{E}$.

Conflicts of Interest: The authors declare no conflict of interest.

$\begin{array}{ll}\text { Abbreviations } \\ \text { CRISPR } & \text { Clustered regularly interspaced short palindromic repeats } \\ \text { Cas } & \text { CRISPR-associated protein } \\ \text { sgRNA } & \text { Single guide RNA } \\ \text { GMOs } & \text { Genetically modified organisms } \\ \text { PAM } & \text { Protospacer adjacent motif } \\ \text { indel } & \text { Insertion and deletion } \\ \text { SNV } & \text { Single nucleotide variation } \\ \text { WGS } & \text { Whole-genome sequencing } \\ \text { NHEJ } & \text { Non-homologous end joining } \\ \text { DSB } & \text { Double-strand break } \\ \text { HDR } & \text { Homology-directed repair }\end{array}$




\section{References}

1. Mojica, F.J.M.; Díez-Villaseñor, C.; García-Martínez, J.; Soria, E. Intervening Sequences of Regularly Spaced Prokaryotic Repeats Derive from Foreign Genetic Elements. J. Mol. Evol. 2005, 60, 174-182. [CrossRef] [PubMed]

2. Alexander, B.; Benoit, Q.; Alexei, S.; Dusko, E. Clustered regularly interspaced short palindrome repeats (CRISPRs) have spacers of extrachromosomal origin. Microbiology 2005, 151, 2551-2561.

3. Jinek, M.; East, A.; Cheng, A.; Lin, S.; Ma, E.; Doudna, J. RNA-programmed genome editing in human cells. eLife 2013, 2, e00471. [CrossRef] [PubMed]

4. Wenyan, J.; David, B.; David, C.; Feng, Z.; Marraffini, L.A. RNA-guided editing of bacterial genomes using CRISPR-Cas systems. Nat. Biotechnol. 2013, 31, 233-239.

5. Dali, L.; Zhongwei, Q.; Yanjiao, S.; Yuting, C.; Yuting, G.; Meizhen, L.; Yongmei, L.; Na, G.; Liren, W.; Xiaoling, L. Heritable gene targeting in the mouse and rat using a CRISPR-Cas system. Nat. Biotechnol. 2013, 31, 681.

6. $\quad$ Platt, R.; Chen, S.; Zhou, Y.; Yim, M.; Swiech, L.; Kempton, H.; Dahlman, J.; Parnas, O.; Eisenhaure, T.; Jovanovic, M. CRISPR-Cas9 Knockin Mice for Genome Editing and Cancer Modeling. Cell 2014, 159, 440-455. [CrossRef]

7. Shan, Q.; Wang, Y.; Li, J.; Zhang, Y.; Chen, K.; Liang, Z.; Zhang, K.; Liu, J.; Xi, J.J.; Qiu, J.L. Targeted genome modification of crop plants using a CRISPR-Cas system. Nat. Biotechnol. 2013, 31, 686-688. [CrossRef] [PubMed]

8. Kim, D.; Alptekin, B.; Budak, H. CRISPR/Cas9 genome editing in wheat. Funct. Integr. Genom. 2018, 18, 31-41. [CrossRef]

9. Feng, C.; Yuan, J.; Wang, R.; Liu, Y.; Birchler, J.A.; Han, F. Efficient Targeted Genome Modification in Maize Using CRISPR/Cas9 System. J. Genet. Genom. 2015, 43, 37-43. [CrossRef]

10. Feng, Z.; Zhang, B.; Ding, W.; Liu, X.; Yang, D.L.; Wei, P.; Cao, F.; Zhu, S.; Zhang, F.; Mao, Y. Efficient genome editing in plants using a CRISPR/Cas system. Cell Res. 2013, 23, 1229-1232. [CrossRef]

11. Wang, H.; La Russa, M.; Qi, L.S. CRISPR/Cas9 in Genome Editing and Beyond. Annu. Rev. Biochem. 2016, 85, 227-264. [CrossRef] [PubMed]

12. Jaganathan, D.; Ramasamy, K.; Sellamuthu, G.; Jayabalan, S.; Venkataraman, G. CRISPR for Crop Improvement: An Update Review. Front. Plant Sci. 2018, 9, 985. [CrossRef] [PubMed]

13. Mishra, R.; Zhao, K. Genome editing technologies and their applications in crop improvement. Plant Biotechnol. Rep. 2018, 12, 57-68. [CrossRef]

14. Wang, F.; Wang, C.; Liu, P.; Lei, C.; Hao, W.; Gao, Y.; Liu, Y.G.; Zhao, K. Enhanced Rice Blast Resistance by CRISPR/Cas9-Targeted Mutagenesis of the ERF Transcription Factor Gene OsERF922. Plos ONE 2016, 11, e0154027. [CrossRef] [PubMed]

15. Shi, J.; Gao, H.; Wang, H.; Lafitte, H.R.; Archibald, R.L.; Yang, M.; Hakimi, S.M.; Mo, H.; Habben, J.E. ARGOS8 variants generated by CRISPR-Cas9 improve maize grain yield under field drought stress conditions. Plant Biotechnol. J. 2017, 15, 207-216. [CrossRef] [PubMed]

16. Waltz, E. Gene-edited CRISPR mushroom escapes US regulation. Nature 2016, 532, 293. [CrossRef] [PubMed]

17. Jinek, M.; Jiang, F.; Taylor, D.W.; Sternberg, S.H.; Kaya, E.; Ma, E.; Anders, C.; Hauer, M.; Zhou, K.; Lin, S.; et al. Structures of Cas9 endonucleases reveal RNA-mediated conformational activation. Science 2014, 343, 1247997. [CrossRef] [PubMed]

18. Kosicki, M.; Tomberg, K.; Bradley, A. Repair of double-strand breaks induced by CRISPR-Cas9 leads to large deletions and complex rearrangements. Nat. Biotechnol. 2018, 36, 765-771. [CrossRef]

19. Zhang, Y.; Ge, X.; Yang, F.; Zhang, L.; Zheng, J.; Tan, X.; Jin, Z.B.; Qu, J.; Gu, F. Comparison of non-canonical PAMs for CRISPR/Cas9-mediated DNA cleavage in human cells. Sci. Rep. 2014, 4, 5405. [CrossRef]

20. Meng, X.; Hu, X.; Liu, Q.; Song, X.; Gao, C.; Li, J.; Wang, K. Robust genome editing of CRISPR-Cas9 at NAG PAMs in rice. Sci. China Life Sci. 2018, 61, 122-125. [CrossRef]

21. Tsai, S.Q.; Nguyen, N.T.; Malagon-Lopez, J.; Topkar, V.V.; Aryee, M.J.; Joung, J.K. CIRCLE-seq: A highly sensitive in vitro screen for genome-wide CRISPR-Cas9 nuclease off-targets. Nat. Methods 2017, 14, 607-614. [CrossRef] 
22. Yang, L.; Grishin, D.; Wang, G.; Aach, J.; Zhang, C.Z.; Chari, R.; Homsy, J.; Cai, X.; Zhao, Y.; Fan, J.B.; et al. Targeted and genome-wide sequencing reveal single nucleotide variations impacting specificity of Cas9 in human stem cells. Nat. Commun. 2014, 5, 5507. [CrossRef]

23. Kleinstiver, B.P.; Pattanayak, V.; Prew, M.S.; Tsai, S.Q.; Nguyen, N.T.; Zheng, Z.; Joung, J.K. High-fidelity CRISPR-Cas9 nucleases with no detectable genome-wide off-target effects. Nature 2016, 529, 490-495. [CrossRef]

24. Slaymaker, I.M.; Gao, L.; Zetsche, B.; Scott, D.A.; Yan, W.X.; Zhang, F. Rationally engineered Cas9 nucleases with improved specificity. Science 2016, 351, 84-88. [CrossRef]

25. Ran, F.A.; Hsu, P.D.; Wright, J.; Agarwala, V.; Scott, D.A.; Zhang, F. Genome engineering using the CRISPR-Cas9 system. Nat. Protoc. 2013, 8, 2281-2308. [CrossRef]

26. Wu, X.; Kriz, A.J.; Sharp, P.A. Target specificity of the CRISPR-Cas9 system. Quant. Biol. 2014, 2, 59-70. [CrossRef]

27. Wu, X.; Scott, D.A.; Kriz, A.J.; Chiu, A.C.; Hsu, P.D.; Dadon, D.B.; Cheng, A.W.; Trevino, A.E.; Konermann, S.; Chen, S.; et al. Genome-wide binding of the CRISPR endonuclease Cas9 in mammalian cells. Nat. Biotechnol. 2014, 32, 670-676. [CrossRef]

28. Zhang, Q.; Xing, H.L.; Wang, Z.P.; Zhang, H.Y.; Yang, F.; Wang, X.C.; Chen, Q.J. Potential high-frequency off-target mutagenesis induced by CRISPR/Cas9 in Arabidopsis and its prevention. Plant Mol. Biol. 2018, 96, 445-456. [CrossRef]

29. Zhao, H.; Wolt, J.D. Risk associated with off-target plant genome editing and methods for its limitation. Emerg. Top. Life Sci. 2017, 1, 231-240. [CrossRef]

30. Kadam, U.S.; Shelake, R.M.; Chavhan, R.L.; Suprasanna, P. Concerns regarding 'off-target' activity of genome editing endonucleases. Plant Physiol. Biochem. 2018, 131, 22-30. [CrossRef]

31. Fu, Y.; Foden, J.A.; Khayter, C.; Maeder, M.L.; Reyon, D.; Joung, J.K.; Sander, J.D. High-frequency off-target mutagenesis induced by CRISPR-Cas nucleases in human cells. Nat. Biotechnol. 2013, 31, 822-826. [CrossRef]

32. Veres, A.; Gosis, B.S.; Ding, Q.; Collins, R.; Ragavendran, A.; Brand,H.; Erdin, S.; Cowan, C.A.; Talkowski, M.E.; Musunuru, K. Low Incidence of Off-Target Mutations in Individual CRISPR-Cas9 and TALEN Targeted Human Stem Cell Clones Detected by Whole-Genome Sequencing. Cell Stem Cell 2014, 15, 27-30. [CrossRef]

33. Feng, Z.; Mao, Y.; Xu, N.; Zhang, B.; Wei, P.; Yang, D.L.; Wang, Z.; Zhang, Z.; Zheng, R.; Yang, L.; et al. Multigeneration analysis reveals the inheritance, specificity, and patterns of CRISPR/Cas-induced gene modifications in Arabidopsis. Proc. Natl. Acad. Sci. 2014, 111, 4632-4637. [CrossRef]

34. Peterson, B.A.; Haak, D.C.; Nishimura, M.T.; Teixeira, P.J.; James, S.R.; Dangl, J.L.; Nimchuk, Z.L. Genome-Wide Assessment of Efficiency and Specificity in CRISPR/Cas9 Mediated Multiple Site Targeting in Arabidopsis. PLoS ONE 2016, 11, e0162169. [CrossRef]

35. Tang, X.; Liu, G.; Zhou, J.; Ren, Q.; You, Q.; Tian, L.; Xin, X.; Zhong, Z.; Liu, B.; Zheng, X.; et al. A large-scale whole-genome sequencing analysis reveals highly specific genome editing by both Cas9 and Cpf1 (Cas12a) nucleases in rice. Genome Biol. 2018, 19, 84. [CrossRef]

36. Li, J.; Manghwar, H.; Sun, L.; Wang, P.; Wang, G.; Sheng, H.; Zhang, J.; Liu, H.; Qin, L.; Rui, H.; et al. Whole genome sequencing reveals rare off-target mutations and considerable inherent genetic or/and somaclonal variations in CRISPR/Cas9-edited cotton plants. Plant Biotechnol. J. 2018, 17, 858-868. [CrossRef]

37. Bae, S.; Park, J.; Kim, J.S. Cas-OFFinder: A fast and versatile algorithm that searches for potential off-target sites of Cas9 RNA-guided endonucleases. Bioinformatics 2014, 30, 1473-1475. [CrossRef]

38. Montague, T.G.; Cruz, J.M.; Gagnon, J.A.; Church, G.M.; Valen, E. CHOPCHOP: A CRISPR/Cas9 and TALEN web tool for genome editing. Nucleic Acids Res. 2014, 42, W401-W407. [CrossRef]

39. Aidan, O.B.; Bailey, T.L. GT-Scan: Identifying unique genomic targets. Bioinformatics 2014, 30, $2673-2675$.

40. Moreno-Mateos, M.A.; Vejnar, C.E.; Beaudoin, J.D.; Fernandez, J.P.; Mis, E.K.; Khokha, M.K.; Giraldez, A.J. CRISPRscan: Designing highly efficient sgRNAs for CRISPR-Cas9 targeting in vivo. Nat. Methods 2015, 12, 982-988. [CrossRef]

41. Tsai, S.Q.; Zheng, Z.; Nguyen, N.T.; Liebers, M.; Topkar, V.V.; Thapar, V.; Wyvekens, N.; Khayter, C.; Iafrate, A.J.; Le, L.P.; et al. GUIDE-seq enables genome-wide profiling of off-target cleavage by CRISPR-Cas nucleases. Nat. Biotechnol. 2015, 33, 187-197. [CrossRef]

42. Kim, D.; Bae, S.; Park, J.; Kim, E.; Kim, S.; Yu, H.R.; Hwang, J.; Kim, J.I.; Kim, J.S. Digenome-seq: Genome-wide profiling of CRISPR-Cas9 off-target effects in human cells. Nat. Methods 2015, 12, 237-243. [CrossRef] 
43. Akcakaya, P.; Bobbin, M.L.; Guo, J.A.; Malagon-Lopez, J.; Clement, K.; Garcia, S.P.; Fellows, M.D.; Porritt, M.J.; Firth, M.A.; Carreras, A.; et al. In vivo CRISPR editing with no detectable genome-wide off-target mutations. Nature 2018, 561, 416-419. [CrossRef]

44. Xing, H.L.; Dong, L.; Wang, Z.P.; Zhang, H.Y.; Han, C.Y.; Liu, B.; Wang, X.C.; Chen, Q.J. A CRISPR/Cas9 toolkit for multiplex genome editing in plants. BMC Plant Biol. 2014, 14, 327. [CrossRef]

45. Qin, G.; Gu, H.; Ma, L.; Peng, Y.; Deng, X.W.; Chen, Z.; Qu, L.J. Disruption of phytoene desaturase gene results in albino and dwarf phenotypes in Arabidopsis by impairing chlorophyll, carotenoid, and gibberellin biosynthesis. Cell Res. 2007, 17, 471-482. [CrossRef]

46. Hsu, P.D.; Scott, D.A.; Weinstein, J.A.; Ran, F.A.; Konermann, S.; Agarwala, V.; Li, Y.; Fine, E.J.; Wu, X.; Shalem, O.; et al. DNA targeting specificity of RNA-guided Cas9 nucleases. Nat. Biotechnol. 2013, 31, 827-832. [CrossRef]

47. Pattanayak, V.; Lin, S.; Guilinger, J.P.; Ma, E.; Doudna, J.A.; Liu, D.R. High-throughput profiling of off-target DNA cleavage reveals RNA-programmed Cas9 nuclease specificity. Nat. Biotechnol. 2013, 31, 839-843. [CrossRef]

48. Zhang, J.H.; Pandey, M.; Kahler, J.F.; Loshakov, A.; Harris, B.; Dagur, P.K.; Mo, Y.Y.; Simonds, W.F. Improving the specificity and efficacy of CRISPR/CAS9 and gRNA through target specific DNA reporter. J. Biotechnol. 2014, 189, 1-8. [CrossRef]

49. Kim, D.; Kim, S.; Kim, S.; Park, J.; Kim, J.S. Genome-wide target specificities of CRISPR-Cas9 nucleases revealed by multiplex Digenome-seq. Genome Res. 2016, 26, 406-415. [CrossRef]

50. Oliveros, J.C.; Franch, M.; Tabas-Madrid, D.; San-Leon, D.; Montoliu, L.; Cubas, P.; Pazos, F. Breaking-Cas-interactive design of guide RNAs for CRISPR-Cas experiments for ENSEMBL genomes. Nucleic Acids Res. 2016, 44, W267-W271. [CrossRef]

51. Pawluk, A.; Amrani, N.; Zhang, Y.; Garcia, B.; Hidalgo-Reyes, Y.; Lee, J.; Edraki, A.; Shah, M.; Sontheimer, E.J.; Maxwell, K.L.; et al. Naturally Occurring Off-Switches for CRISPR-Cas9. Cell 2016, 167, 1829-1838. [CrossRef]

52. Oakes, B.L.; Fellmann, C.; Rishi, H.; Arkin, A.P.; Doudna, J.A.; Savage, D.F. CRISPR-Cas9 Circular Permutants as Programmable Scaffolds for Genome Modification. Cell 2019, 176, 256-267. [CrossRef]

53. Shou, J.; Li, J.; Liu, Y.; Wu, Q. Precise and Predictable CRISPR Chromosomal Rearrangements Reveal Principles of Cas9-Mediated Nucleotide Insertion. Mol. Cell 2018, 71, 498-509. [CrossRef]

54. Kim, J.M.; Kim, D.; Kim, S.; Kim, J.S. Genotyping with CRISPR-Cas-derived RNA-guided endonucleases. Nat. Commun. 2014, 5, 3157. [CrossRef]

55. Wang, Z.P.; Xing, H.L.; Dong, L.; Zhang, H.Y.; Han, C.Y.; Wang, X.C.; Chen, Q.J. Egg cell-specific promoter-controlled CRISPR/Cas9 efficiently generates homozygous mutants for multiple target genes in Arabidopsis in a single generation. Genome Biol. 2015, 16, 144. [CrossRef]

56. Martin, M. Cutadapt removes adapter sequences from high-throughput sequencing reads. Embnet. J. 2011, 17, 10-12. [CrossRef]

57. Langmead, B.; Salzberg, S.L. Fast gapped-read alignment with Bowtie 2. Nat. Methods 2012, 9, 357-359. [CrossRef]

58. McKenna, A.; Hanna, M.; Banks, E.; Sivachenko, A.; Cibulskis, K.; Kernytsky, A.; Garimella, K.; Altshuler, D.; Gabriel, S.; Daly, M.; et al. The Genome Analysis Toolkit: A MapReduce framework for analyzing next-generation DNA sequencing data. Genome Res. 2010, 20, 1297-1303. [CrossRef]

59. Wilm, A.; Aw, P.P.K.; Bertrand, D.; Yeo, G.H.T.; Ong, S.H.; Wong, C.H.; Khor, C.C.; Petric, R.; Hibberd, M.L.; Nagarajan, N. LoFreq: A sequence-quality aware, ultra-sensitive variant caller for uncovering cell-population heterogeneity from high-throughput sequencing datasets. Nucleic Acids Res. 2012, 40,11189-11201. [CrossRef]

60. Cibulskis, K.; Lawrence, M.S.; Carter, S.L.; Sivachenko, A.; Jaffe, D.; Sougnez, C.; Gabriel, S.; Meyerson, M.; Lander, E.S.; Getz, G. Sensitive detection of somatic point mutations in impure and heterogeneous cancer samples. Nat. Biotechnol. 2013, 31, 213-219. [CrossRef]

61. Koboldt, D.C.; Zhang, Q.; Larson, D.E.; Shen, D.; McLellan, M.D.; Lin, L.; Miller, C.A.; Mardis, E.R.; Ding, L.; Wilson, R.K. VarScan 2: Somatic mutation and copy number alteration discovery in cancer by exome sequencing. Genome Res. 2012, 22, 568-576. [CrossRef] 
62. Ye, K.; Schulz, M.H.; Long, Q.; Apweiler, R.; Ning, Z. Pindel: A pattern growth approach to detect break points of large deletions and medium sized insertions from paired-end short reads. Bioinformatics 2009, 25, 2865-2871. [CrossRef]

63. Rausch, T.; Zichner, T.; Schlattl, A.; Stutz, A.M.; Benes, V.; Korbel, J.O. DELLY: Structural variant discovery by integrated paired-end and split-read analysis. Bioinformatics 2012, 28, i333-i339. [CrossRef]

(C) 2019 by the authors. Licensee MDPI, Basel, Switzerland. This article is an open access article distributed under the terms and conditions of the Creative Commons Attribution (CC BY) license (http://creativecommons.org/licenses/by/4.0/). 